\title{
Automated Assessment of Left Ventricular Function and Mass Using Heart Deformation Analysis: Initial Experience in 160 older adults
}

\author{
Kai Lin, MD, MS ${ }^{1}$, Jeremy D. Collins, MD¹, Donald M. Lloyd-Jones, MD, MS², Marie-Pierre \\ Jolly, PhD ${ }^{3}$, Debiao Li, PhD ${ }^{1,{ }^{*}}$, Michael Markl, PhD ${ }^{1}$, and James C. Carr, MD1 \\ ${ }^{1}$ Department of Radiology, Northwestern University, 737 N Michigan Avenue, Suite 1600, \\ Chicago, IL 60611 \\ ${ }^{2}$ Department of Preventive Medicine, Northwestern University, 680 N Lake shore drive, Suite \\ 1400, Chicago, IL 60611 \\ ${ }^{3}$ Siemens Corporation, Corporate Technology, 755 College Road East, Princeton, NJ 08540
}

\begin{abstract}
Objective-To assess the performance of automated quantification of left ventricular function and mass based on heart deformation analysis (HDA) in asymptomatic older adults

Materials and methods-This study complied with HIPAA regulations. Following the approval of the institutional review board (IRB), 160 asymptomatic older participants were recruited for cardiac MRI including two-dimensional (2D) cine images covering the entire left ventricle (LV) in short-axis view. Data analysis included the calculation of left ventricular ejection fraction (LVEF), mass (LVM) and cardiac output (CO) using HDA and standard global cardiac function analysis (delineation of end systolic and diastolic LV epi- and endo-cardial borders). The agreement between methods was evaluated using intra-class correlation coefficient (ICC) and coefficient of variation $(\mathrm{CoV})$.
\end{abstract}

Results-HDA had a shorter processing time than standard method ( $1.5 \pm 0.3$ minute/case vs. $5.8 \pm 1.4$ minute/case, $\mathrm{p}<0.001$ ). There was good agreement for $\mathrm{LVEF}(\mathrm{ICC}=0.552, \mathrm{CoV}=$ $10.5 \%), \mathrm{CO}(\mathrm{ICC}=0.773, \mathrm{CoV}=13.5 \%)$ and $\mathrm{LVM}(\mathrm{ICC}=0.859, \mathrm{CoV}=14.5 \%)$ acquired with standard method and HDA. There was a systemic bias towards lower LVEF $(62.8 \% \pm 8.3 \%$ vs. $69.3 \% \pm 6.7 \%, \mathrm{p}<0.001)$ and $\mathrm{CO}(4.4 \pm 1.0 \mathrm{~L} /$ minute vs. $4.8 \pm 1.3 \mathrm{~L} /$ minute, $\mathrm{p}<0.001)$ by HDA compared to the standard technique. Conversely, HDA overestimated LVM $(114.8 \pm 30.1 \mathrm{~g}$ vs. $100.2 \pm 29.0 \mathrm{~g}, \mathrm{p}<0.001$ ) as compared to the reference method.

\footnotetext{
Address for correspondence: Kai Lin, kai-lin@ @orthwestern.edu, Department of Radiology, Northwestern University, 737 N Michigan Avenue, Suite 1600, Chicago, IL 60611, Tel: (312)695-5577, Fax: (312)926-5991.

* Current address: Cedars Sinai Medical Center, 8700 Beverly Blvd., Los Angeles, CA 90048

Financial disclosure:

A co-author, MPJ, is employee of SIEMENS AG. The data and information of this study are under control by authors who are not SIEMENS employee.

Publisher's Disclaimer: This is a PDF file of an unedited manuscript that has been accepted for publication. As a service to our customers we are providing this early version of the manuscript. The manuscript will undergo copyediting, typesetting, and review of the resulting proof before it is published in its final citable form. Please note that during the production process errors may be discovered which could affect the content, and all legal disclaimers that apply to the journal pertain.
} 
Conclusion-HDA has the potential to measure LVEF, CO, and LVM without the need for user interaction based on standard cardiac 2D Cine images.

\section{Keywords}

Heart deformation analysis; MRI; left ventricular function; mass

\section{Introduction}

Cardiac aging, which can result in subclinical alterations in the heart, is considered a condition that bridges elderly and the incidence of cardiovascular events. At the same time, co-existing cardiovascular confounders, such as diabetes mellitus (DM) and hypertension (HTN), can further contribute to the development of cardiac aging. Left ventricular (LV) ejection fraction (LVEF) and mass (LVM) are indices presenting ventricular dysfunction and hypertrophy, two important indicators in cardiovascular risk estimation. In clinical practice, two-dimensional (2D) cine magnetic resonance imaging (MRI) has been adopted as a standard method for the measurement of LVEF and LVM. Balanced steady-state free processing (b-SSFP) is a common used MRI technique for the acquisition of cine MRI images at short-axis view because it provides good blood-myocardium contrasts (1). Generally, endocardial and epicardial borders of the LV are determined automatically or manually for each slice/phase of MRI based on signal differences among blood pool, LV wall and outer structures. LV volumes at each cardiac phase will be obtained by summing up the LV areas in all slices covering the heart from the base to the apex. End-systole and end-diastole can be identified to define the smallest and largest LV volumes, respectively, for calculating LVEF and cardiac output (CO). LV myocardial volume will be calculated by adding up the LV areas in each slice at end-diastole and end-systole. LVM can therefore be calculated by assuming a reasonable myocardial density.

Heart deformation analysis (HDA) is a recently developed imaging processing technique for the description of global and regional myocardial function and motion on cine MRI. Using a deformation image registration (DIR) algorithm, HDA is able to automatically track the shape of LV through the entire cardiac cycle by calculating deformation fields (forward and backward) of myocardium tissue among sequential cardiac time frames (2, 3). As such, global cardiac indices (LVEF, CO and LVM) as well as regional myocardial motion indices (displacement, velocity, strain and strain rate) can be extracted from cine images using a semi-automatic "one-stop-shop" analysis with minimal user interaction. However, to date the capability of HDA tool for the evaluation of LV global function and morphology has not been validated in a larger clinical study cohort. Therefore, the aim of the present study was to assess the accuracy of automated quantification of left ventricular function and mass based on HDA in asymptomatic older adults by using a standard LV global function analysis as the reference standard. 


\section{Materials and Methods}

\section{Study participants}

This study complied with HIPAA regulations. Following the approval of the institutional review board (IRB), 160 asymptomatic older participants of the Chicago Healthy Aging Low-Risk Magnetic Resonance Angiography Study (CHARISMA), including 93 male subjects (mean age $71.8 \pm 4.3$ years old, range $66-84$ years old) and 67 female subjects (mean age $72.4 \pm 3.9$ years old, range $65-83$ years old), were recruited and underwent cardiac cine MRI (4). All participants did not have documented history of cardiovascular disease at the time of MRI. Written informed consent was provided by all participants. See table 1 for the description of the study cohort.

\section{MRI facility}

For all participants in this study, the MRI examinations were performed by 2 certified clinical MRI technicians on a $1.5 \mathrm{~T}$ scanner (SIEMENS, MAGNATOM, Espree, Germany) with a 6-channel cardiac phased-array coil.

\section{Cine MRI scan protocol}

A 3-plane, rapid localization sequence was run first to provide anatomic orientation for the entire scan. A black-blood half-Fourier acquisition single-shot turbo spin-echo sequence was used to obtain images using standard 4-chamber and short-axis display orientations. A 2dimentional (2D) b-SSFP sequence was used to take cardiac cine images with a 2-chamber view and 4-chamber view of the heart.

Then, the 2D CINE b-SSFP sequence was used to acquire cardiac cine images (22 retrospectively reconstructed cardiac phases) at the short-axis view of the heart with ECG triggering. Two transverse LV slices were acquired within a breath-hold at the end of expiration. Five or six breath-hold scans ( $10-12$ slices) were acquired to cover the entire heart from base to apex. Imaging parameters included: TR/TE $=2.8 / 1.1 \mathrm{msec}$; flip angle $=$ $65^{\circ}$, voxel size $=2.1 \times 2.1 \times 6.0 \mathrm{~mm}^{3}$, parallel acquisition factor $=2$.

\section{Image processing}

Standard cine image processing-All images were reviewed by experienced staff of our imaging core lab. Images that were not eligible for quantitative analysis were excluded. Stacks of cardiac cine images in the short-axis were analyzed using a commercial software (Siemens Argus, Leonardo Syngo workstation, clinical device with FDA 510(k) clearance). Endocardial and epicardial borders were automatically detected by the software based on signal differences between blood and myocardium. Two independent observers, who were blinded to the HDA results, fine-tuned the LV contours for each cine images interactively. Papillary muscles were regularly excluded in the $\mathrm{LV}$ wall area during myocardial border drawing because papillary and trabecular volumes can only generate a small difference for the calculation of cardiac function (5). LVEF was calculated as follows: LVEF $=($ SV/EDV) $* 100$. Where SV = stroke volume = EDV $-\mathrm{ESV}, \mathrm{EDV}=$ end-diastolic volume, ESV = endsystolic volume. $\mathrm{CO}=\mathrm{SV} *$ heart rate $(\mathrm{HR})$. The total $\mathrm{LV}$ myocardial volume was defined 
as the summation of LV volumes for all slices. LVM was calculated as: LV volume * myocardial density (set as $1.05 \mathrm{~g} / \mathrm{ml}$ ).

HDA analysis-Cine MRI images acquired at multiple imaging planes, including 2chamber, 4-chamber and a stack of short-axis images were loaded to a dedicated image processing workstation (Dell, STUDIO, SPS 435T) and analyzed with prototype software programmed in Visual C++ (TrufiStrain, Siemens Corporation, Princeton, NJ) by an experienced reader (_, with 10 years of experience in cardiovascular imaging) who was blinded to the results of previous function analysis. The HDA tool automatically detects anatomical landmarks in the heart as anchor points to initialize the automatic detection of the myocardial borders, including the mitral valve and apex point at the long-axis views, aortic valve anchor points and the right ventricle [RV] insertion/lateral points at the shortaxis views. Using an existing DIR algorithm, elastic image registration was completed to calculate frame-to-frame motion deformation fields (2). A 2D displacement vector was then assigned to each pixel within specific deformation area. To register two adjacent time frames and obtain 2D displacement vectors, the DIR algorithm can minimize the local cross correlation between the displaced pixels on the moving image and the reference image, while enforcing the deformation fields of myocardial structure to stay smooth. Between any two cardiac time frames on each LV slice, gradient descent minimization was applied to compute a dense deformation field. All cardiac phases were segmented using a shortest path algorithm and time consistency is enforced through the deformation fields (3). A multiseeded fuzzy connectedness (MFC) algorithm was used to discriminate tissues of lung, myocardium and blood by grouping pixels according to gray levels (3). The myocardial borders for each frame were then delineated based on the deformation areas. Finally, LVEF, $\mathrm{CO}$ and LVM (averaged over values measured at end-diastole and end-systole, assuming myocardial density $=1.05 \mathrm{~g} / \mathrm{ml}$ ) could be generated using the myocardial borders as for Argus (6). In addition, the mitral valve base plane is detected in the long axis slices (using the mitral valve landmark points) and intersected with the short axis contours to remove the atrial portions from the endocardial and epicardial volumes.

Reader \#1 reviewed all HDA-generated LV contours. Cine images with poor image quality could result in "unreasonable" contours (defined as contours significantly including non-LV structures) and would be excluded for analysis. No manually adjustments were imposed on HDA-generated contours.

Since contour drawing and landmark selection were performed fully automatically during HDA analysis, no operator input was required for analysis. It was thus not necessary to test the intra- or inter-observer variations of HDA. Reader \#1 reloaded cine images of 10 randomly chosen participants a week later and repeated the same workflow to test the interanalysis variability.

\section{Data processing and statistical analysis}

All continuous variables were represented as mean \pm one standard deviation (SD). LVEF, $\mathrm{CO}$ and LVM measured by HDA and the standard method (clinical reference standard) were compared using paired $t$-tests. The intra-class correlation coefficient (ICC) and coefficient of 
variation ( $\mathrm{CoV}$, defined as $\mathrm{SD} / \mathrm{Mean})$ were applied to describe the agreement of peak myocardial measurements acquired from two consecutive scans. An ICC value $>0.75$ or a $\mathrm{CoV}<20 \%$ between duplicated measurements was considered as good agreement. BlandAltman plots were used to visualize the inter-method variances of individual myocardial motion indices. Statistical analysis was performed using SPSS statistical software (Version 13.0, SPSS. Inc, Chicago, IL). All statistical analyses were two-tailed. A $p$ value $<0.05$ was considered statistically significant.

\section{Results}

Nine 2D cine b-SSFP datasets were excluded for either standard method or HDA (judged by the core lab staff and reader \#1, respectively) due to poor image quality and 151 datasets were finally included. Five participants had low LVEF (defined as $<50 \%$, measured with the standard method). Figure 1 shows a typical dataset that was analyzed using two methods.

HDA has shorter processing time than standard method ( $1.5 \pm 0.3$ minute/case vs. $5.8 \pm 1.4$ minute/case, $\mathrm{p}<0.001$ ). There was good agreement between LVEF $(\mathrm{ICC}=0.552, \mathrm{CoV}=$ $10.5 \%), \mathrm{CO}(\mathrm{ICC}=0.773, \mathrm{CoV}=13.5 \%)$ and $\mathrm{LVM}(\mathrm{ICC}=0.859, \mathrm{CoV}=14.5 \%)$ acquired with the standard method and HDA. See figure 2 for the Bland-Altman plots of inter-method variations between the two approaches.

Despite of good agreement between two methods, we also found that there were significant differences in LV function and mass between the two techniques (see table 2). Specially, HDA underestimated LVEF $(62.8 \% \pm 8.3 \%$ vs. $69.3 \% \pm 6.7 \%, \mathrm{p}<0.001)$ and CO $(4.4 \pm 1.0$ $\mathrm{L} /$ minute vs. $4.8 \pm 1.3 \mathrm{~L} /$ minute, $\mathrm{p}<0.001)$ compared to the standard method. In addition, HDA was prone to overestimate LVM $(114.8 \pm 30.1 \mathrm{~g}$ vs. $100.2 \pm 29.0 \mathrm{~g}, \mathrm{p}<0.001)$ compared to the standard method.

Reanalysis of 10 2D Cine b-SSFP datasets by reader \#1 (without manual inputs that will affect the measures of LV function and LVM) yielded identical results compared to the first analysis.

\section{Discussion}

The findings of our study demonstrate the potential of HDA for the quantification of left ventricular global function and mass. High ICC and low CoV clearly indicated good agreement of LVEF, CO and LVM measured with HDA compared to the clinical reference standard in 151 older adults. Our results suggest that HDA has the potential to serve as an alternative to provide comparable cardiac function for cardiovascular risk estimation, especially for asymptomatic elderly, a major target population for cardiovascular prevention.

Both decreased LVEF and increased LVM are reliable predictors of outcome of cardiovascular diseases. Currently, echocardiography and MRI are two major noninvasive imaging methods for measuring LVEF and LVM. However, there is not a consensus regarding the "best choice" for measuring LVEF and LVM. For echocardiography, different imaging facilities and different formula for the calculation of LVEF and LVM can result in 
diverse values (7). With higher inter-study reproducibility, MRI seems to be a more reliable imaging method for evaluating LVEF and LVM (8).

HDA was developed for describing myocardial motion/deformation based on MRI images using DIR algorithms. DIR algorithm has been applied to calculate myocardial strains or lung motion in previous studies $(9,10)$. Different from existing method for tracking cardiac motion by identifying edge-, or speckle-like characteristics of a structure, such as feature tracking (FT), HDA tool can automatically define and calculate deformation fields for the myocardial structures, including LV wall and blood-pool, over time frames through the entire cardiac cycle $(2,3,11)$. With an operator-independent workflow, HDA tool is expected to serve as a robust tool that generates multiple indices for the comprehensive description of myocardial function, motion and morphological changes. This advantage is valuable in clinical study because multiple myocardial indices form various aspects are necessary for differential diagnosis of similar cardiovascular disorders. Using the same cardiac cine datasets, our data demonstrated that HDA-derived LVEF, CO and LVM are comparable to those LV values measured with existing method.

Despite correlations between LV measures acquired with two methods, we also noticed that HDA may overestimate or underestimate LV functional and morphological indices. In theory, symmetry and inverse consistency are two major principles for DIR algorithms in analyzing series medical images, such as cine MRI. Different from the methods used for ARGUS, our HDA tool used 6 processing steps to segment myocardial tissue in the LV and calculate deformation fields for the acquisition of myocardial motion indices, including polar space transformation, gray scale analysis, deformable registration, gradient computation, Dijkstra's shortest path access, and final contour recovery (3). Base on high myocardium-blood pool contrast demonstrated on cine images, HDA is able to accurately identify LV borders. However, the difference in the volume calculation with the addition of the base planes may serve as a major source for the disagreement between ARGUS and HDA. HDA used the long-axis images (at 2- or 4-chamber views) to discriminate LV and left atrium (LA). While the operator manually exclude LA tissue at base levels of LV for the calculation of LVEF, LVM and CO. For most 2D imaging techniques, including echocardiography and computed tomography (CT), selection of imaging planes can significantly affect the measures of cardiac function and mass $(12,13)$. As a result, the existence of systemic bias for measuring LVEF and LVM among between standard clinical methods is not a surprise (14). For example, M-mode echocardiography, a 2D technique which needs manual selection of appropriate imaging planes, seems to systematic overestimate LVM as compared with transesophageal 3-dimensional echocardiography (3DE) and MRI (7). Furthermore, the LVEF values obtained with echocardiography, angiography, radionuclide ventriculography (RNV), and MRI are not fully interchangeable in recipients of heart transplantation (HTx) (15).

Our study has limitations. First, we did not include patients with diagnosed cardiovascular diseases since the participants of CHARISMA study were asymptomatic elderly. The aim of the present study is not to assess the capability of the HDA for the detection of low LVEF. Actually, our study cohort included only 5 subjects with low LVEF $(<50 \%)$. Additional studies are required and warranted to evaluate the performance of HDA in describing 
cardiac function in special patient groups. Second, we did not evaluate the accuracy of the HDA tool in measuring other myocardial motion indices in the present study. Due to the fixed MRI protocol in CHARISMA study, dedicated MRI sequences for tracking myocardial motion, such as tagging, $D$ isplacement $e n$ coding with stimulated $e$ choes (DENSE) or tissue phase mapping (TPM) could not be added to the scans. Third, we were unable to predict cardiovascular events in those participants using HDA-derived LVEF, CO and LVM because of the small sample size. However, a follow-up study has been initiated to evaluate the roles of the HDA-derived myocardial function and motion indices in cardiovascular risk estimation.

In conclusion, HDA has the potential to measure LVEF, CO, and LVM without the need for user interaction based on standard cardiac 2D Cine images.

\section{Acknowledgments}

This study was supported by three grants from the National Institute of Health (R01HL089695, R01HL117888 and K01HL121162).

\section{List of abbreviations}

$\begin{array}{ll}\text { HDA } & \text { Heart deformation analysis } \\ \text { MRI } & \text { Magnetic resonance imaging } \\ \text { LVEF } & \text { Left ventricular ejection fraction } \\ \text { LVM } & \text { Left ventricular mass } \\ \text { CO } & \text { cardiac putput }\end{array}$

\section{References}

1. Tyler DJ, Hudsmith LE, Petersen SE, et al. Cardiac cine MR-imaging at 3T: FLASH vs SSFP. Journal of cardiovascular magnetic resonance: official journal of the Society for Cardiovascular Magnetic Resonance. 2006; 8(5):709-15. [PubMed: 16891230]

2. Guetter C, Xue H, Chefd'hotel C, et al. Efficient symmetric and inverse-consistent deformable registration through interleaved optimization. Biomedical Imaging: From Nano to Macro, 2011 IEEE International Symposium on. 2011:590-593.

3. Jolly M-P, Guetter C, Lu X, Xue H, Guehring J. Automatic Segmentation of the Myocardium in Cine MR Images Using Deformable Registration. Statistical Atlases and Computational Models of the Heart Imaging and Modelling Challenges. 2012; 7085:98-108.

4. Pirzada A, Reid K, Kim D, et al. Chicago Healthy Aging Study: objectives and design. Am J Epidemiol. 2013; 178(4):635-44. [PubMed: 23669655]

5. Sievers B, Kirchberg S, Bakan A, Franken U, Trappe HJ. Impact of papillary muscles in ventricular volume and ejection fraction assessment by cardiovascular magnetic resonance. Journal of cardiovascular magnetic resonance: official journal of the Society for Cardiovascular Magnetic Resonance. 2004; 6(1):9-16. [PubMed: 15054924]

6. MacDonald KA, Kittleson MD, Reed T, Larson R, Kass P, Wisner ER. Quantification of left ventricular mass using cardiac magnetic resonance imaging compared with echocardiography in domestic cats. Veterinary radiology \& ultrasound: the official journal of the American College of Veterinary Radiology and the International Veterinary Radiology Association. 2005; 46(3):192-9.

7. Kuhl HP, Bucker A, Franke A, et al. Transesophageal 3-dimensional echocardiography: in vivo determination of left ventricular mass in comparison with magnetic resonance imaging. Journal of 
the American Society of Echocardiography: official publication of the American Society of Echocardiography. 2000; 13(3):205-15. [PubMed: 10708469]

8. Bottini PB, Carr AA, Prisant LM, Flickinger FW, Allison JD, Gottdiener JS. Magnetic resonance imaging compared to echocardiography to assess left ventricular mass in the hypertensive patient. American journal of hypertension. 1995; 8(3):221-8. [PubMed: 7794570]

9. Phatak NS, Maas SA, Veress AI, Pack NA, Di Bella EV, Weiss JA. Strain measurement in the left ventricle during systole with deformable image registration. Medical image analysis. 2009; 13(2): 354-61. [PubMed: 18948056]

10. Boldea V, Sharp GC, Jiang SB, Sarrut D. 4D-CT lung motion estimation with deformable registration: quantification of motion nonlinearity and hysteresis. Medical physics. 2008; 35(3): 1008-18. [PubMed: 18404936]

11. Schuster A, Kutty S, Padiyath A, et al. Cardiovascular magnetic resonance myocardial feature tracking detects quantitative wall motion during dobutamine stress. Journal of cardiovascular magnetic resonance: official journal of the Society for Cardiovascular Magnetic Resonance. 2011; 13:58. [PubMed: 21992220]

12. Gottdiener JS, Bednarz J, Devereux R, et al. American Society of Echocardiography recommendations for use of echocardiography in clinical trials. Journal of the American Society of Echocardiography: official publication of the American Society of Echocardiography. 2004; 17(10):1086-119. [PubMed: 15452478]

13. Schlosser T, Pagonidis K, Herborn CU, et al. Assessment of left ventricular parameters using 16MDCT and new software for endocardial and epicardial border delineation. AJR American journal of roentgenology. 2005; 184(3):765-73. [PubMed: 15728595]

14. Seo HY, Lee SP, Park JB, et al. Discrepancies in Left Ventricular Mass Calculation Based on Echocardiography and Cardiovascular Magnetic Resonance Measurements in Patients with Left Ventricular Hypertrophy. Journal of the American Society of Echocardiography: official publication of the American Society of Echocardiography. 2015

15. Bellenger NG, Marcus NJ, Rajappan K, Yacoub M, Banner NR, Pennell DJ. Comparison of techniques for the measurement of left ventricular function following cardiac transplantation. Journal of cardiovascular magnetic resonance: official journal of the Society for Cardiovascular Magnetic Resonance. 2002; 4(2):255-63. [PubMed: 12074140] 

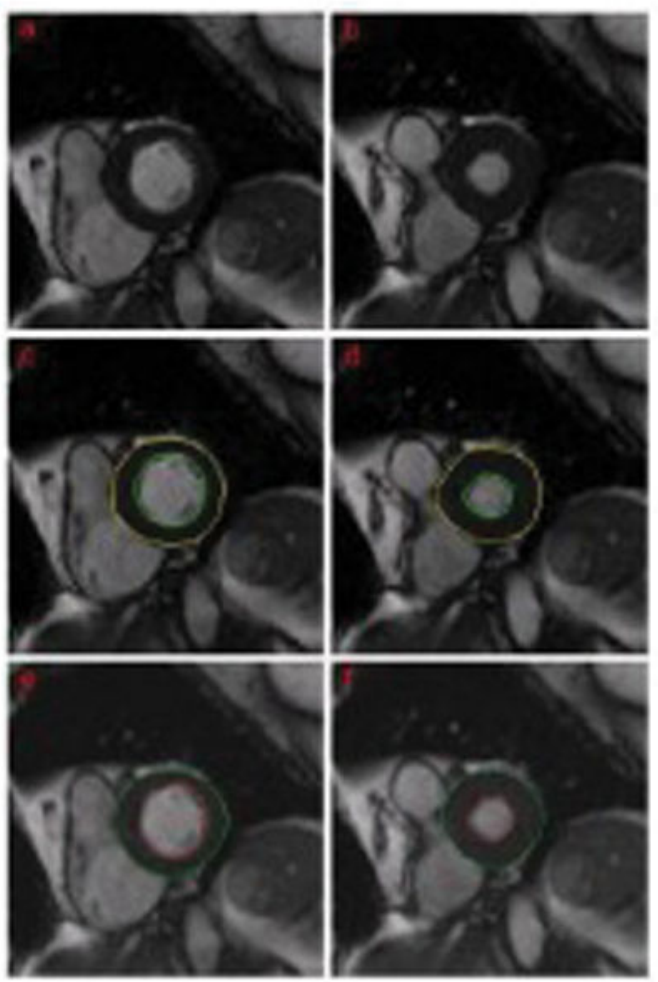

\section{Figure 1.}

A 83 years old female with HTN. The 2D cine b-SSFP images were analyzed using HDA and the standard method. Papillary muscles were regularly excluded.

a An original LV image (short-axial view) at diastole.

b An original LV image (short-axial view) at systole.

c LV wall (at diastole) defined by calculating deformation fields with DIR algorithm.

$\mathrm{d} L \mathrm{~V}$ wall (at systole) defined by calculating deformation fields with DIR algorithm. The $\mathrm{LVEF}=63.6 \%, \mathrm{CO}=4.3 \mathrm{~L} /$ minute, and $\mathrm{LVM}=113.8 \mathrm{~g}$.

e LV wall (at diastole) defined with ARGUS at our imaging core.

$\mathrm{f} \mathrm{LV}$ wall (at systole) defined with ARGUS at our imaging core. The LVEF $=68.4 \%, \mathrm{CO}=$ $4.6 \mathrm{~L} /$ minute, and $\mathrm{LVM}=98.1 \mathrm{~g}$. 

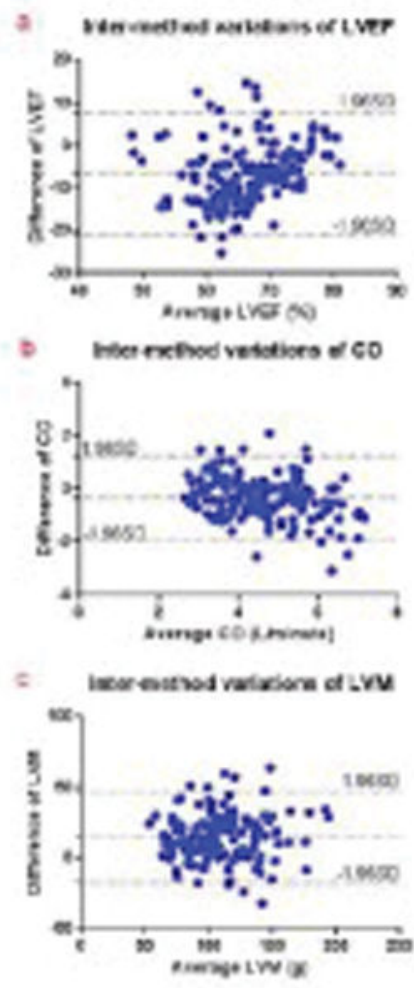

Figure 2.

Bland-Altman plots show inter-method variations of LVEF, CO and LVM measures $(\mathrm{n}=$ 151).

a Inter-method variations of LVEF

$\mathrm{b}$ Inter-method variations of $\mathrm{CO}$

c Inter-method variations of LVM 


\section{Table 1}

\begin{tabular}{|c|c|c|}
\hline \multicolumn{3}{|c|}{ Description of participants } \\
\hline \multirow{9}{*}{ 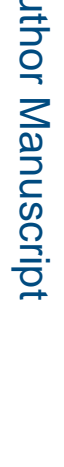 } & & Subjects $(N=160)$ \\
\hline & Male (\%) & $97(60)$ \\
\hline & Age (years old) & $72.0 \pm 4.1$ \\
\hline & Weight $(\mathrm{kg})$ & $86.3 \pm 17.8$ \\
\hline & Height (cm) & $172.1 \pm 9.8$ \\
\hline & BMI & $28.9 \pm 5.8$ \\
\hline & Heart rate (beats/minute) & $69.7 \pm 10.2$ \\
\hline & $\mathrm{DM}(\%)$ & $39(24)$ \\
\hline & $\operatorname{HTN}(\%)$ & $57(36)$ \\
\hline
\end{tabular}

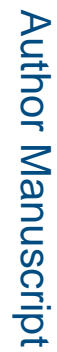

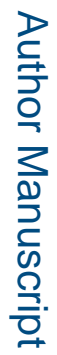

D

Acad Radiol. Author manuscript; available in PMC 2017 March 01. 
Table 2

The differences of LVEF, CO amd LVM measured with the HDA tool and existing method (using paired $t$ tests).

\begin{tabular}{|l|l|l|l|l|}
\hline & Processing time (minutes) & LVEF $(\%)$ & CO (L/minute) & LVM (g) \\
\hline HDA & $1.5 \pm 0.3$ & $62.8 \pm 8.3$ & $4.4 \pm 1.0$ & $114.8 \pm 30.1$ \\
\hline Reference method & $5.8 \pm 1.4$ & $69.3 \pm 6.7$ & $4.8 \pm 1.3$ & $100.2 \pm 29.0$ \\
\hline$p$ values & $<0.001$ & $<0.001$ & $<0.001$ & $<0.001$ \\
\hline
\end{tabular}

